\title{
Méthode larvoscopique quantitative et qualitative utilisant des boîtes fermées pour le diagnostic et l'étude de certaines Helminthiases vétérinaires
}

\author{
par Paul DANCESCO
}

Chaire de Parasitologie, Faculté de Perfectionnement des médecins, Bucarest, Roumanie

\begin{abstract}
Résumé
La méthode présentée est modifiée d'après la technique de culture sur charbon dans des boîtes fermées (Dancesco et coll. 1967) et utilisée dans le diagnostic de la strongylö̈dose humaine. Cette dernière méthode a été elle-même inspirée par la méthode française de culture sur charbon en forme de cône (Sarrazin et coll. 1962, Carré 1968). La méthode décrite dans le présent travail diffère de celles mentionnées plus haut, par le fait que l'on n'utilise plus la poudre de charbon. Il en résulte une appréciation quantitative ou de l'intensité parasitaire plus exacte.

En plus l'examen n'est plus pratiqué en retournant le couvercle de la boîte, mais au contraire à travers le couvercle. On évite ainsi des variations dans le volume des gouttes de condensation dues à l'évaporation; par suite, on peut noter des observations de durée plus exactes sur la biologie et les réactions des larves aux facteurs physiques.
\end{abstract}

\section{Summary}

This method is a modified technic from the French coproculture method (Sarrazin \& coll., 1962; Carré, 1968) and the culture on coal in closed boxes of Dancesco \& coll. (1967). The coal powder is no longer used and this allows a more valuable approach of the density of parasitism and of the number of larvae.

Moreover, the larvae are directly observed through the coverbox in spite of turning it over before inspection. So there are few variations in drops of condensation due to evaporation, and time for evolution or larval reactions to physical factors are more easily observed. 


\section{Matériel.}

- Boîtes en matière plastique transparente, de $1,7-2 \mathrm{~cm}$ de hauteur, $5 \mathrm{~cm}$ de diamètre, $28 \mathrm{ml}$ capacité. On peut utiliser des boîtes type médicaments.

- Baguettes de roseau ou de verre.

- Crayon dermographique.

- Microscope stéréoscopique.

\section{Technique de travail.}

Des déjections récoltées aseptiquement sont placées sous forme de cône dans une boîte en matière plastique transparente. Lorsque la boîte est recouverte, la pointe du cône doit atteindre le centre du couvercle formant une tache de contact d'approximativement $1 \mathrm{~cm}^{2} \emptyset$, visible de l'extérieur. La boîte est gardée fermée, à l'ombre, à la température de la pièce, soit $16^{\circ}-25^{\circ} \mathrm{C}$. Des gouttes de condensation se forment sous le couvercle pendant 24 heures, persistent et augmentent les jours suivants. Les larves qui se trouvent dans les déjections ou celles qui éclosent s'élèvent, grâce à leur géotropisme négatif, vers la pointe du cône, et traversent la pellicule de liquide autour de la tache centrale sous le couvercle s'amassant dans les gouttes de condensation (hygrotropisme). De là les larves ont tendance à migrer d'une manière centrifuge vers les gouttes de condensation alentour.

L'examen s'effectue à travers le couvercle, en gardant la boîte fermée, à l'aide d'un microscope stéréoscopique (loupe binoculaire), avec l'objectif $4 \mathrm{X}$ et l'oculaire 6,3 X. La lumière doit arriver en faisceau latéral, à angle aigu par rapport au plan de la platine du microscope. Dans ces conditions il est évident que pas une seule larve n'échappe à l'observation.

On note le résultat en comptant les larves ou en évaluant le nombre de gouttes contenant des larves. On note aussi le jour où les larves sont apparues.

Etant donné qu'on peut compter les larves sous le couvercle de la boîte, et calculer par la différence de poids entre la boîte contenant la préparation et la boîte vide, le poids des déjections qui ont été introduites (en moyenne de 5-9 grammes), le résultat peut être exprimé par le nombre de larves par gramme de selles.

En vue de l'identification des larves, le couvercle de la boîte est soulevé et appliqué sur une autre boîte vide ; elles sont ensuite examinées au microscope à l'objectif $10 \mathrm{X}$ ou $20 \mathrm{X}$ en relevant le condenseur. On observe aisément la structure interne des larves. En vue d'une étude plus détaillée, les larves peuvent être retirées et montées dans des préparations pour les conserver.

En maintenant pendant un certain temps les boîtes fermées on peut noter quelques observations sur la biologie des larves, leur longévité et autres données.

On peut poursuivre ces observations biologiques pendant plusieurs semaines ou mois sans traumatiser les larves. 


\section{Application de la méthode.}

On présente, dans les lignes qui suivent, les premiers résultats de l'application de cette méthode, utilisée pour préciser la fréquence du parasite Strongyloides papillosus ainsi que pour l'étude de la bio'ogie des larves appartenant à différentes espèces de Nématodes parasites du mouton. La méthode nous a permis d'observer:

1. - La fréquence globale des helminthiases et le moment de l'apparition des premières larves appartenant aux différentes espèces de Nématodes. D'un lot de 39 moutons examinés, 94,6\% ont présenté des larves dans les déjections, plus de 24 heures après la préparation. Deux fois seulement, les larves ont été dépistées le jour même de la préparation.

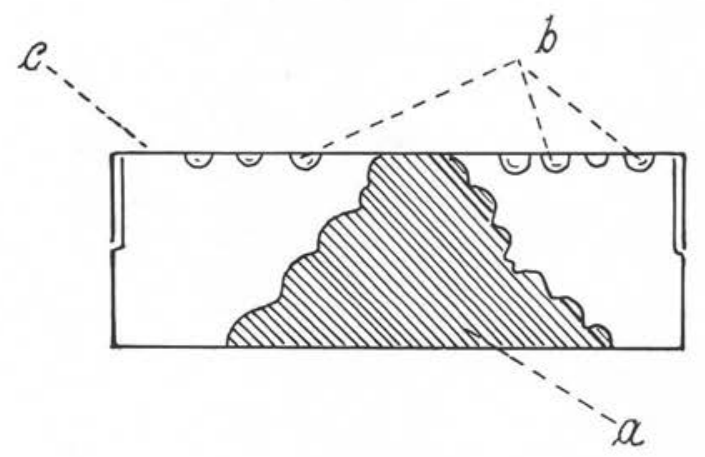

Fig. 1. - Schéma de la boîte fermée pour larvoscopie: $a$ ) déjections animales; b) gouttes de condensation ; c) couvercle transparent

2. - Fréquence de Strongyloides papillosus. Les larves ont été identifiées dans $87,2 \%$ des échantillons. On remarque facilement, avec l'objectif $10 \mathrm{X}$ du microscope, les détails suivants :

- l'œsophage de type rhabditoïde ;

- le rapport entre la longueur de l'œsophage et celle du corps de la larve ;

- l'anneau nerveux périœsophagien ;

— l'extrémité caudale qui est légèrement bifide; parfois, on observe une troisième proéminence médiane punctiforme ;

- les larves décrivent dans leur mouvement des sinuosités de deux et demi (dans les gouttes d'eau volumineuses, stables), à trois et demi demi-cercles (généralement dans les gouttes éclatées, aplaties) (fig. 4).

3. - Le moment de l'apparition des adultes de l'espèce S. papillosus et leur longévité. Les adultes ont été observés rarement et en nombre réduit à partir du troisième jour. Ils vivent $24,48,72$ heures dans les gouttes de condensation. La deuxième génération de larves n'a pas été obtenue, étant donné que les rares adultes étaient isolés dans les gouttes d'eau. 


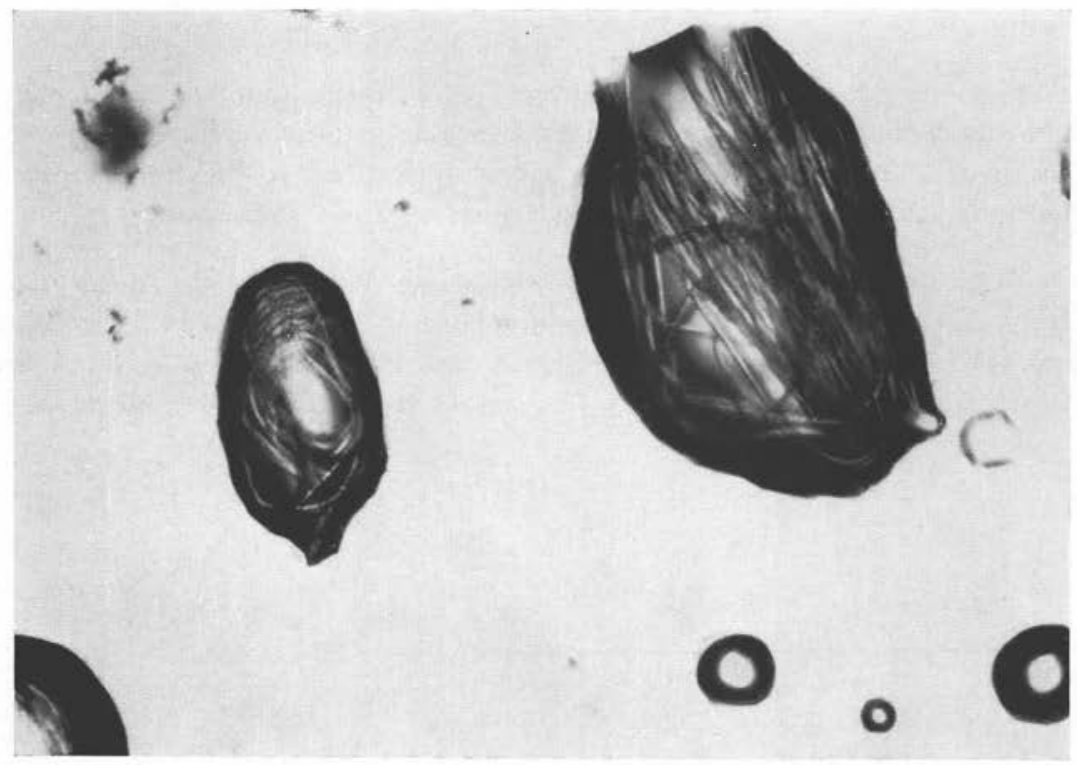

FIG. 2

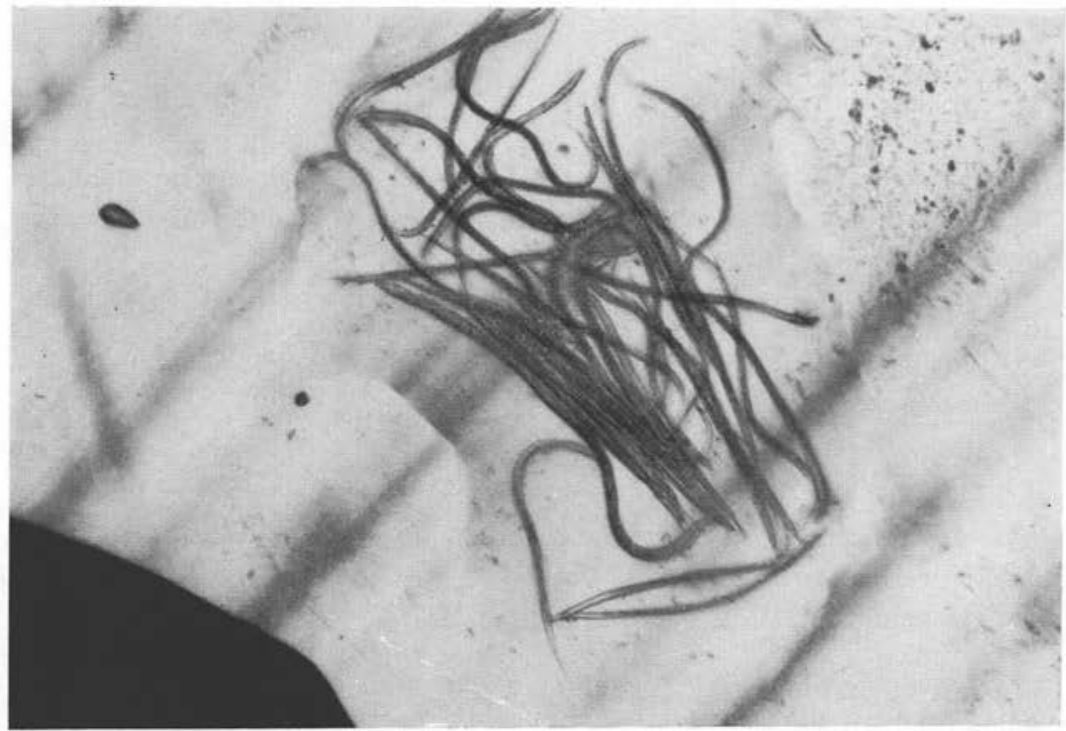

FIG. 3

FIG. 2 et 3. - Strongyloides papillosus. Culture en boîtes fermées (microphotographie originale) 
4. - La longévité des larves appartenant à différentes espèces: l'évolution des larves s'est poursuivie pendant 73 jours. La courbe des échantillons positifs monte rapidement (fig. 5). Les larves présentent de forts mouvements déclenchés surtout par un faisceau lumineux ou par la charge électrique réalisée en frottant légèrement le couvercle de la boîte avec le doigt. Au fur et à mesure que les préparations vieillissent les mouvements diminuent en intensité. On a noté le moment où la totalité des larves d'une boîte sont mortes. Après 40 jours la courbe des échantillons avec des larves vivantes commence à décliner. Vu le nombre important des espèces de parasites du mouton dont on peut trouver les larves dans les déjections, la différence entre les diverses courbes de longévité spécifique n’a pu être établie.
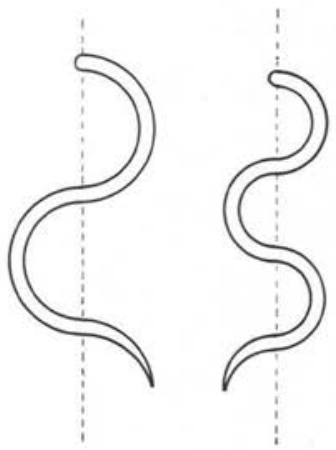

Fig. 4. - Strongyloides papillosus. Les mouvements des larves. a) Généralement dans les gouttes d'eau volumineuses et stables. b) Généralement dans les gouttes d'eau éclatées et aplaties, ou en dehors des gouttes quand les larves ont une fine pellicule d'eau autour du corps

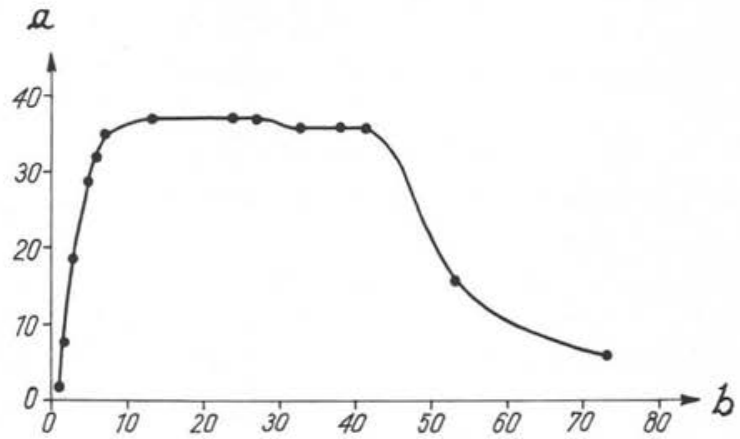

FIg. 5. - La courbe de la longévité des larves appartenant à différentes espèces de Nématodes parasites chez le mouton. Préparation en boite fermée. a) Nombre d'échantillons positifs; $b$ ) nombre de jours après la préparation

\section{Discussion.}

La technique de travail est très simple et facile à exécuter. Les déjections sont placées dans des boîtes sur le lieu même de la récolte. On peut récolter quelques centaines d'épreuves en un seul jour.

Etant donné que les boîtes sont petites, incassables, légères, de taille uniforme et parfaitement closes, elles peuvent être aisément manœuvrées et d'autre part l'examen au microscope stéréoscopique est facile et rapide; on peut contrôler quelques centaines d'examens journellement. Le matériel de travail est bon marché et on élimine le nettoyage en remplaçant la verrerie par des récipients en matériel non-récupérable. On peut effectuer les préparations facilement sur le terrain, et le transport des boîtes 
au laboratoire peut avoir lieu dans de bonnes conditions dans n'importe quel véhicule, à quelque distance que ce soit, pendant plusieurs jours.

La méthode supprime les temps morts et réduit le personnel au travail.

\section{Bibliographie}

1. CARRÉ (S.), 1968. — La strongyloïdose humaine parasitose d'importation. Thèse. Faculté de Médecine de Toulouse.

2. Dancesco (P.) et Radulesco (S.), 1967. - Microbiol. Parasitol. Epidemiol. (Bucarest), 12,1 , pp. $35-40$.

3. -, 1968. - Bull. Soc. Path. exot., 61, 4, pp. 651-661.

4. Ho-Thi-Sang, Brumpt (L.) et Lapierre (J.), 1951. - Bull. Soc. Path. exot., 54, 5, pp. 1059 1075.

5. - 1966. - Communication personnelle.

6. Niculescu (A.) et Lungu (T. R.), 1961. - Travaux pratiques de Parasitologie. Ed. Inst. Agron. Bucarest.

7. Sarrazin (A.), Gentilini (M.) et Deparis (M.), 1962. - Concours méd. 22, pp. 3.385 3.390 .

8. Lapierre (J.), 1969. - Evolution Médicale, 13, 1, pp. 19-25. 